\title{
LE PATRIMOINE COMME LEVIER DE DÉVELOPPEMENT TERRITORIAL. LE CAS DES RESSOURCES PATRIMONIALES DU PARC NATIONAL DE TLEMCEN (ALGÉRIE)
}

\author{
Yamina NECISSA, Naima CHABBI CHEMROUK
}

\begin{abstract}
Résumé
En Algérie, la protection et la mise en valeur du patrimoine est l'une des orientations stratégiques du Schéma National d'Aménagement du Territoire à l'horizon 2030. À cette fin, les Pôles d'Économie du Patrimoine et les Parcs Culturels sont introduits comme une nouvelle alternative de développement territorial articulé autour d'un concept nouveau : " le projet de territoire ». Or, la mise en œuvre de ce projet dépend non seulement de facteurs physiques spécifiques au patrimoine en question mais surtout de l'implication effective de toutes les parties prenantes. Les acteurs locaux qui sont confrontés au quotidien, aux besoins de leurs territoires sont souvent exclus d'un processus de décision caractérisé par une forte centralisation.

Par conséquent, l'objectif du présent article consiste à élaborer et tester un outil d'évaluation de la capacité de contribution des ressources patrimoniales au développement durable territorial. Le résultat attendu est de proposer une démarche de mise en valeur des projets patrimoniaux basée sur un diagnostic des différentes potentialités et contraintes et sur l'ouverture du processus de prise de décision aux acteurs locaux et aux habitants. Le parc national de Tlemcen dans la région Ouest de l'Algérie a été sélectionné comme cas d'étude au vu de la richesse de son patrimoine paysager et culturel et de par l'échelle intercommunale qu'il couvre.
\end{abstract}

Mots-clés

analyse multicritère, territoire, diagnostic, ressources patrimoniales

\begin{abstract}
In Algeria, the protection and valorization of heritage is one of the strategic orientations of the National Land Use Planning Plan for 2030. In this plan, Heritage Economy Poles and Cultural Parks are introduced as a new alternative for territorial development articulated around a new concept: "the territorial project". However, the implementation of this project depends not only on the physical characteristics of the heritage in question but also on the effective implication of all the stakeholders. Local actors who are confronted to every day's needs are very often absent from the decision-making process.

Therefore, the objective of this article is to develop and test a tool for assessing the capacity of heritage resources to sustainable territorial development. The expected result is to propose an approach to enhance heritage projects based on a diagnosis of the different potentials and constraints and on the inclusion of local actors as well as inhabitants in the decision-making process.

The Tlemcen National Park in the western region of Algeria is used as a demonstrative case study, as it is characterized by the richness of its landscape and cultural heritage and by the inter-municipal scale that it covers.
\end{abstract}

Keywords

multi-criteria analysis, territory, diagnosis, heritage resources

\section{INTRODUCTION}

La valorisation des ressources patrimoniales a souvent été introduite pour améliorer l'attractivité touristique de certains territoires et booster le tourisme (Kancel et al., 2013 ; Vernieres, 2015). Ainsi, beaucoup de destinations touristiques comme Athènes, Rome, Londres et Paris ont souvent misé sur la valorisation des ressources patrimoniales comme levier de développement de l'industrie touristique. Au-delà de l'attrait touristique, les ressources culturelles, naturelles et paysagères sont désormais de plus en plus mobilisées dans des projets plus inclusifs : « des projets de territoires » (François et al., 2006).

Dans ce sens, cet article part du postulat que la valorisation du patrimoine ne doit pas être une fin en soi. Elle doit être intégrée dans un programme de développement territorial. En tant que ressource, le patrimoine culturel, naturel et paysager mobilisé dans les démarches de développement des territoires pourra donner à chacun d'eux une vocation propre, une personnalité. En effet, comme 
le souligne Guérin (2001), « le patrimoine, parce qu'il se réfère aux héritages, crée la personnalité du territoire ».

À cet égard, au-delà des politiques de développement économique et d'aménagement du territoire en cours, la nouvelle démarche de développement territorial mise sur la spécificité de chaque territoire particulièrement celle liée à son patrimoine qu'elle cherche à mobiliser (Baudelle et al., 2011). Cependant, la valorisation et l'intégration des ressources patrimoniales dans un programme de développement territorial est un processus assez complexe à mettre en place. Ce processus ne se restreint pas à un listing d'objectifs aussi clairs et bien circonscrits soient-ils. Il s'agit d'identifier et d'inventorier les ressources patrimoniales existantes, d'en évaluer la capacité à être intégrées dans des projets à travers notamment les plans généraux, mais aussi d'identifier les acteurs aussi bien publics que privés capables de veiller au développement territorial souhaité et imaginé par les populations de ces territoires.

Le présent article s'appuie sur une revue de littérature sur les ressources patrimoniales en lien avec le projet de territoire ainsi que sur les orientations de la stratégie nationale d'aménagement du territoire en Algérie, pour élaborer un outil d'évaluation de la contribution des ressources patrimoniales au développement territorial. Le résultat attendu est celui d'aboutir à une stratégie de mise en valeur du patrimoine et d'orienter la prise de décision des acteurs dans le cadre de projets du développement territorial.

\section{MÉTHODES ET OUTILS}

L'élaboration d'une grille multicritère qui permettrait d'évaluer la contribution des ressources patrimoniales au développement territorial doit s'inscrire dans la vision des orientations du schéma national d'aménagement et de développement territorial. Pour répondre à cet objectif, une première étape consacrée à la lecture analytique de ces orientations était donc nécessaire.

Par ailleurs, le diagnostic des ressources patrimoniales est essentiel à la compréhension du contexte dont lequel l'outil d'évaluation sera vérifié. Plusieurs travaux ont montré que le diagnostic reste une étape incontournable dans la construction des scénarii et autres propositions de développement
(François et al., 2013). Il permet aussi d'identifier les objet-acteurs susceptibles d'être mobilisés dans le cadre de la construction de la ressource territoriale (Senil, 2011).

Dans ce cas particulier, le diagnostic consiste à étudier la dimension territoriale des ressources patrimoniales (accessibilité, typologies et autres caractéristiques, acteurs en charge de la gestion des ressources, etc.). L'objectif est d'identifier les ressources qui pourraient contribuer au développement du territoire concerné ainsi que les acteurs impliqués et leurs stratégies. L'inventaire comme outil conventionnel d'identification des biens culturels a donc été utilisé pour répertorier les objets patrimoniaux présents sur le territoire.

Le diagnostic a été complété par la réalisation d'entretiens semi-directifs auprès des principaux acteurs en charge de la valorisation du patrimoine. La Figure 1 schématise la démarche et outils utilisés.

\section{ORIENTATIONS STRATÉGIQUES EN MATIÈRE D'AMÉNAGEMENT ET DE DÉVELOPPEMENT TERRITORIAL EN ALGÉRIE}

L'identification et la préservation des ressources ainsi que leur potentiel de valorisation en matière d'aménagement et de développement du territoire est clairement énoncé dans le nouveau Schéma National d'Aménagement du Territoire (SNAT) à l'horizon 2030. Différents textes de loi et en particulier, la loi 01-02 affirme cette volonté de protéger, sauvegarder et valoriser les biens culturels, historiques et archéologiques (JO $\left.\mathrm{N}^{\circ} 77,2001, \mathrm{p} .15\right)$.

Par ailleurs, cette volonté de valorisation se retrouve au niveau des quatre lignes directrices établies pour la mise en œuvre du SNAT :

- la ligne directrice $n^{\circ} 1:$ La durabilité des ressources ;

- la ligne directrice $n^{\circ} 2:$ Le rééquilibrage du territoire ;

- la ligne directrice $n^{\circ} 3:$ L'attractivité et la compétitivité du territoire ;

- la ligne directrice $n^{\circ} 4: 1^{\prime}$ 'équité sociale et territoriale ;

La première ligne directrice du SNAT vise la durabilité du territoire en établissant ainsi un lien étroit entre aménagement du territoire et durabilité et en 
Le cas des ressources patrimoniales du parc national de Tlemcen (Algérie)

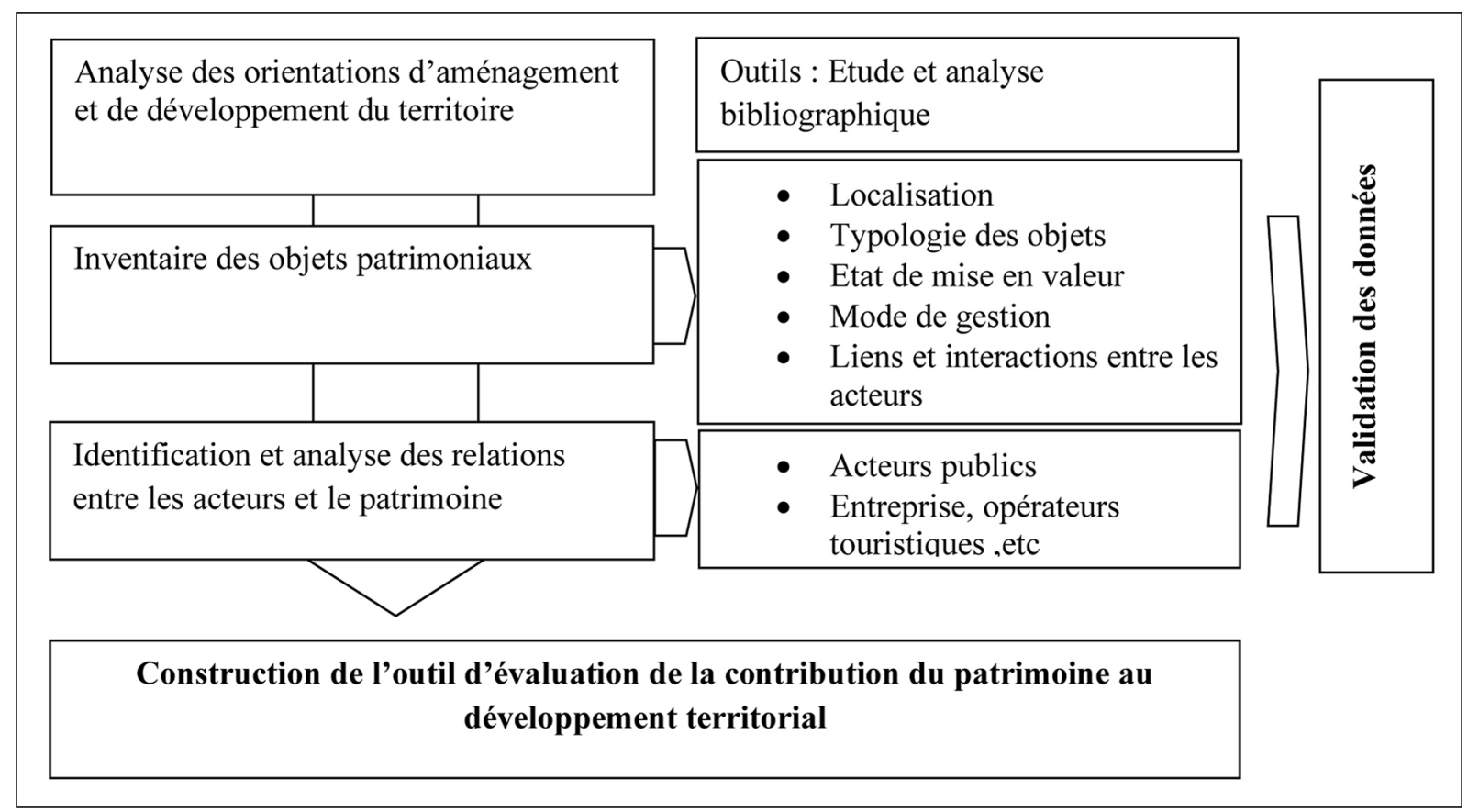

Figure 1. Schéma de la démarche suivie. Source : Necissa, 2020

faisant de celle-ci une préoccupation préalable à toute intervention dans le domaine de l'aménagement.

L'approche territoriale du développement durable mise en particulier sur la préservation du capital naturel et culturel, la valorisation économique et sociale de ce capital, notamment dans le cadre d'une politique de développement local.

La conciliation des impératifs de la préservation des biens culturels protégés et les exigences du développement économique est recommandée à travers les orientations du schéma directeur des zones archéologiques et historiques. Le diagnostic effectué lors de l'étude du SNAT a ainsi permis l'identification des pôles patrimoniaux ayant une grande valeur matérielle et immatérielle. Ils sont identifiés comme parcs culturels, secteurs sauvegardés et sites historiques et archéologiques (loi 10-02, 2010, p.40). L'objectif étant la protection et la mise en valeur du patrimoine national dans une stratégie générale d'aménagement et de développement du territoire.

Les effets attendus de cette valorisation sur le développement sont de plusieurs ordres » (loi 10-02, 2010, p.11) :

- le développement du tourisme et des loisirs ;

- le développement d'un secteur d'économie culturelle directement lié à la valorisation du patrimoine ;
- le renforcement de l'attractivité et de la notoriété du territoire.

La place du patrimoine semble relativement importante dans la stratégie d'aménagement et de développement territorial. L'étude et l'analyse des orientations de cette stratégie sont nécessaires pour l'évaluation de la contribution du patrimoine au développement territorial.

\section{CONSTRUCTION DE L'OUTIL D'ÉVA- LUATION}

La collecte d'informations s'est principalement effectuée via une revue de littérature contribuant à l'établissement des éléments formant la grille multicritère de l'outil d'évaluation (Gautier, 2005 ; Vanderpooten, 2009). Cet outil a l'avantage d'aboutir à une évaluation basée sur des critères multisectoriels ce qui est nécessaire vu la diversité des principes directeurs d'aménagement et de développement.

Les thèmes et les critères d'évaluation de la capacité du patrimoine à être mobilisé dans le processus de développement du territoire découlent des orientations de développement proposés dans le cadre du schéma national d'aménagement et de développement durable du territoire (Loi 10-02, 2010).

La construction de la grille d'analyse multicritère se base sur trois étapes. Dans un premier temps, 
il est nécessaire de rassembler des thèmes, des critères d'évaluation et des énoncés ou indicateurs encadrant ces critères. La deuxième étape permet de déterminer un mode d'évaluation. La dernière étape est consacrée à la pondération des critères d'évaluation.

\section{A. Construction de la grille multicritères avec référence aux principes et orientations de développement du SNAT à l'horizon 2030}

Tout en se référant à la stratégie de préservation et de valorisation du patrimoine fondée sur le plan d'aménagement du territoire pour l'an 2030, quatre orientations de développement du territoire ont été exploitées pour construire les éléments de la grille multicritère.

\section{La protection et la valorisation des aires} protégées et leur évaluation et intégration comme facteur de développement durable des territoires

Les insuffisances de la gestion environnementale montrent la faible prise de conscience des problèmes de protection et de mise en valeur de ce patrimoine. Il s'agit d'une problématique nouvelle pour l'Algérie, ce qui nécessite une véritable mutation intellectuelle et politique, permettant de définir les enjeux propres à chaque espace. La création de onze parcs nationaux, de cinq réserves naturelles et de quatre réserves de chasses offre un potentiel significatif sur plus de $22 \%$ du territoire national (Tableau 1).

2. La protection et la mise en valeur du patrimoine culturel national dans une stratégie générale d'aménagement et de développement du territoire

Selon le SNAT, l'organisation spatiale du système patrimonial doit faire ressortir la distinction entre l'aménagement de l'espace géographique, qui colle à la réalité d'un découpage administratif et la fabrication permanente du territoire par les hommes porteurs d'identités et des cohésions sociales.

C'est en réponse à cet objectif que des pôles patrimoniaux hautement significatifs par leurs valeurs matérielles et immatérielles ont été identifiés et traduits sous la forme de parcs culturels, de secteurs sauvegardés et de sites historiques et archéologiques. Dans la nouvelle configuration politique et stratégique, les propositions de pôles d'économie du patrimoine culturel (PEP), s'établissent autour des secteurs sauvegardés, sites et monuments historiques classés et répartis à l'échelle nationale. Il s'agit d'une nouvelle configuration spatiale à signification socioculturelle qui est une opportunité à l'échelle locale et à la fabrication du territoire (Loi 10-02, 2010).

\section{L'attractivité et la compétitivité des territoires}

Il s'agit de la création des conditions de la compétitivité et de l'attractivité des territoires. Pour atteindre cet objectif, le SNAT propose différentes actions à savoir : la production et l'échange des savoirs faire, l'investissement, la modernisation des infrastructures de transport, la création de plateformes de communication et le rééquilibrage des territoires par la création de pôles de compétitivité et d'excellence.

\section{L'équité sociale et territoriale par la promotion des systèmes productifs, des activités et des emplois}

Parmi les actions proposées par le SNAT : la réduction des inégalités spatiales de développement concentrant les moyens sur les territoires les moins dotés en ressources. La mise en œuvre de ces actions est soutenue par des mesures multiples d'aides financières appropriées et consolidées par le recours au partenariat national et international et par la mise en place d'instances de concertation territoriale et

\begin{tabular}{|l|l|l|}
\hline Aires protégées & Superficies \\
\hline Parcs nationaux & $\begin{array}{l}\text { El Kala, Gouraya, Taza, Theniet el had, Djurdjura, Chrea, } \\
\text { Thniet, Belezma, Tlemcen, Tassili, Ahaggar, Djbelaissa (11) }\end{array}$ & 53193637 ha \\
\hline Réserves naturelles & $\begin{array}{l}\text { la Masta, Mergueb, Beni saleh, Babor, Marins des îles habiba } \\
(5)\end{array}$ & 40283 ha \\
\hline Réserves de chasse & Djelfa, Tlemcen, Mascara, Zeralda (4) & 42200 ha \\
\hline Surface totale & 532275922 ha \\
\hline
\end{tabular}

Tableau 1. Aires protégées en Algérie. Source : Necissa, 2020 
Le cas des ressources patrimoniales du parc national de Tlemcen (Algérie)

la formation des moyens humains nécessaires à l'ingénierie territoriale.

\section{B. Les critères et indicateurs proposés pour l'évaluation}

Les orientations de la politique d'aménagement et de développement durable du territoire ont permis de définir trois thèmes à partir desquels, nous avons élaboré des critères qualitatifs d'évaluation posés sous formes de questions. Par la suite, nous avons évalué la capacité du patrimoine à être mobilisé dans le processus de développement territorial.

\section{Thème 1 : Développement économique}

Le projet patrimonial doit constituer une source d'investissement qui peut engendrer des bénéfices rentables pour l'ensemble du territoire. Le critère d'évaluation issu de ce thème est le suivant : « Le patrimoine est-il utilisé de façon rentable de manière à contribuer au développement économique?».

\section{Thème 2 : Développement social et amélioration de la qualité de vie}

Le patrimoine doit être utilisé de façon à permettre une équité sociale et une amélioration du cadre de vie des citoyens. Le critère d'évaluation proposé est le suivant : «Le projet patrimonial permet-il une qualité de vie meilleure pour la société ? Assure-t-il une solidarité sociale?».

\section{Thème 3 : Gouvernance et gestion du patrimoine}

La protection du patrimoine doit s'inscrire dans une logique de développement durable (les projets de valorisations doivent assurer la transmission du patrimoine aux générations futures).

Dans le but de rapprocher les décisions des citoyens et intégrer les préoccupations sociales, le processus décisionnel de planification et de gestion du patrimoine doit être réalisé en consultant le public.

Il est aussi nécessaire que la mise en valeur du patrimoine soit menée en parfaite collaboration entre les instances décisionnelles à différents niveaux (horizontal et vertical). Deux critères d'évaluation sont proposés : « Le projet patrimonial s'inscrit-il dans une bonne gouvernance ?» et « Le projet pa- trimonial permet-il la conservation et la protection du patrimoine à long terme ? ».

Pour chaque critère, nous avons proposé différents indicateurs répondant aux thèmes proposés. Vanderpooten (2009) définit un critère comme une fonction qui permet réellement à un point de vue donné et pour un acteur identifié de comparer deux actions. Il doit permettre de mesurer les préférences du décideur vis-à-vis de chaque action, relativement à un point de vue. Un indicateur est généralement défini comme un paramètre, ou valeur dérivée d'un paramètre, une variable qualitative ou quantitative qui donne des informations sur un phénomène. C'est une information choisie, associée à un critère, destinée à en observer les évolutions à intervalles définis. Son rôle principal est de réduire le nombre de paramètres normalement nécessaires pour rendre compte d'une situation et simplifier l'interprétation des résultats pour l'utilisateur.

La pertinence des critères et des indicateurs a été vérifiée en concertation avec les acteurs interrogés (chacun selon son domaine). La grille ainsi obtenue couvre 33 indicateurs organisés en trois thèmes majeurs : développement économique, développement social et gestion et gouvernance.

L'ensemble des indicateurs liés à chacun des critères d'évaluation est présenté dans le Tableau 2.

\section{Pondération des indicateurs}

Les critères choisis sont notés selon un système de pondération qualitatif choisi de manière concertée en collaboration avec divers acteurs (Doré, 2005). Dans notre cas, nous avons interrogé les différentes catégories d'acteurs autour de notre objet d'études : responsables territoriaux (acteurs politiques, économiques, administratifs et techniques), qui sont concernés par le développement et la gestion du patrimoine du parc de Tlemcen.

Nous avons choisi une échelle de mesure qualitative se situant entre -3 et +3 . Elle est organisée de la manière suivante : $\bullet$ Mal pris en compte $; \cdot$ Non pris en compte; $\bullet$ Moyennement pris en compte ; -Assez bien pris en compte $; \bullet$ Bien pris en compte.

Le choix du mode de présentation sous forme de radar permet une lecture immédiate et la compa- 


\begin{tabular}{|c|c|c|}
\hline Thèmes & Critères & Énoncés indicateurs \\
\hline 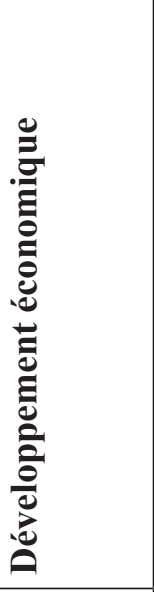 & $\begin{array}{l}\text { Le patrimoine est-il } \\
\text { utilisé de façon } \\
\text { rentable de manière } \\
\text { à contribuer au déve- } \\
\text { loppement écono- } \\
\text { mique? }\end{array}$ & $\begin{array}{l}\text { 1. Attractivités du territoire pour ses activités dans le domaine du patrimoine ; } \\
\text { 2. Activités économiques en place liées au patrimoine : droits d'entrée, trans- } \\
\text { port touristique ; } \\
\text { 3. Dépenses publiques associées au patrimoine ; } \\
\text { 4. Dépenses d'entrée des résidents dans le patrimoine ; } \\
\text { 5. Dépenses de maintenance et d'entretien pour l'accueil des visiteurs ; } \\
\text { 6. Investissements dans l'accueil des visiteurs ; } \\
\text { 7. Activité touristique sur le territoire : entrées, nuitées, dépenses moyennes, } \\
\text { durée de séjour ; } \\
\text { 8. Activité des sites culturels : droits d'entrée, activités annexes ; } \\
\text { 9. Coût de la gestion touristique pour la collectivité : accueil, sécurité, infor- } \\
\text { mation, services, entretien déchets ; } \\
\text { 10. Retombées économiques sur le territoire ; } \\
\text { 11. Équilibre financier de la mise en valeur du patrimoine. }\end{array}$ \\
\hline 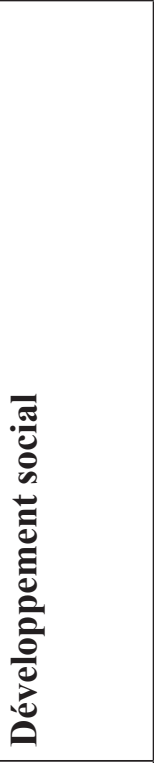 & $\begin{array}{l}\text { Le projet patrimonial } \\
\text { permet-il une qualité } \\
\text { de vie meilleure pour } \\
\text { la société et assure- } \\
\text { t-il une solidarité } \\
\text { sociale? }\end{array}$ & $\begin{array}{l}\text { 1. Personnels spécialisés dans la conservation/restauration du patrimoine } \\
\text { (architectes, artisans, entreprises); } \\
\text { 2. Participation des populations locales aux travaux d'entretien ou de réhabi- } \\
\text { litation/conservation du patrimoine ; } \\
\text { 3. Implication des associations dans la mise en valeur du patrimoine ; } \\
\text { 4. Programmations de réunions annuelles destinées à l'information et/ou la } \\
\text { prise de décision sur la mise en valeur du patrimoine ; } \\
\text { 5. Formations professionnelles dans le domaine du patrimoine ; } \\
\text { 6. Visites scolaires annuelles sur site ; } \\
\text { 7. Organisation des manifestations culturelles liées au patrimoine (festivals } \\
\text { folkloriques, manifestations...) ; } \\
\text { 8. Dépenses destinées à l'organisation de manifestations culturelles liées au } \\
\text { patrimoine (festivals folkloriques, manifestations...); } \\
\text { 9. Dépenses de communication visant à sensibiliser la population locale au } \\
\text { patrimoine ; } \\
\text { 10. Participation de la population locale au processus de prise de décision sur } \\
\text { le patrimoine; } \\
\text { 11. Emplois liés aux dépenses du visiteur. }\end{array}$ \\
\hline 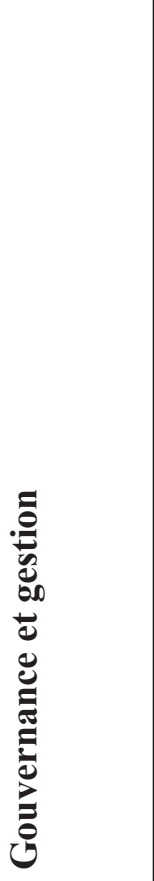 & $\begin{array}{l}\text { Le projet patrimonial } \\
\text { s'inscrit-il dans une } \\
\text { bonne gouvernance? } \\
\text { Permet-il la conser- } \\
\text { vation et la protec- } \\
\text { tion du patrimoine à } \\
\text { long terme? }\end{array}$ & $\begin{array}{l}\text { 1. Existence d'une législation de protection du patrimoine ; } \\
\text { 2. Existence de structures locales, nationales, internationales de prise en charge } \\
\text { de la valorisation du Patrimoine ; } \\
\text { 3. Capacité à mobiliser des aides techniques et financière internationales. } \\
\text { Expériences antérieures ; } \\
\text { 4. Existence de dispositifs financiers publics (locaux, nationaux, internatio- } \\
\text { naux) d'aide à la conservation/valorisation du patrimoine ( Aides directes, } \\
\text { aides indirectes); } \\
\text { 5. Existence de dispositifs financiers privés facilitant le financement de la } \\
\text { conservation/valorisation du patrimoine ; } \\
\text { 6. Existence de dispositifs fiscaux favorisant la valorisation du patrimoine ; } \\
\text { 7. Degré d'autonomie des collectivités locales dans la prise en charge du } \\
\text { patrimoine ; } \\
\text { 8. Labellisation/appartenance du patrimoine (en totalité ou en partie) à un } \\
\text { réseau national ou international du type patrimoine mondial ; } \\
\text { 9. Existence et disponibilité des données - Des médias et des activités d'inter- } \\
\text { prétation mettent en valeur l'histoire et le patrimoine local. (brochure, carte } \\
\text { ou plan, promenade guidée, affiche, site Web); } \\
\text { 10. Le projet s'intègre-t-il dans un plan d'aménagement global : référence } \\
\text { possible à un PDAU ou à un SRAT? } \\
\text { 11. Le projet fait il l'objet d'un diagnostic patrimonial? }\end{array}$ \\
\hline
\end{tabular}

Tableau 2. Énoncés encadrant les critères d'évaluation. Source : Necissa, 2020 
Le cas des ressources patrimoniales du parc national de Tlemcen (Algérie)

raison du niveau de performance de chaque critère et d'identifier visuellement où sont les forces et faiblesses du projet évalué.

\section{CAS D'ÉTUDE : TLEMCEN, LA « GRE- NADE AFRICAINE "}

Ancienne capitale du Maghreb central du XIII ${ }^{\mathrm{e}}$ au IV e siècle (Marçais, 1950 ; Khalifa, 2011) Tlemcen, que certains auteurs qualifient de la « Grenade africaine $^{1} \gg$ (Matait, 2010) est parmi les wilayas les plus riches en potentiel patrimonial (Sari, 2006 ; Gomar, 2007). La wilaya concentrerait le plus grand pourcentage du parc archéologique national en matière de monuments historiques islamiques, c'est dans ce sens, qu'elle a été sélectionnée pour représenter la culture Islamique en 2011 (Khalifa, 2011).

En 2011, en tant que capitale de la culture islamique, Tlemcen a donc concentré pratiquement l'ensemble des subventions de l'État pour mettre en valeur ses sites et monuments historiques. Cette effervescence même conjoncturelle qui caractérise généralement "l'évènementiel » en tant qu'alternative ponctuelle de « city branding » et marketing a favorisé l'intégration de certains sites historiques dans les politiques de développement urbain de la wilaya.

Ainsi, différents sites et monuments ont bénéficié d'opérations plus ou moins réussies de restauration, rénovation ou de réhabilitation. Notre choix s'est porté sur le Parc national de Tlemcen car ce dernier avait été choisi par les autorités locales mais aussi nationales comme projet étendard (Flagship) pour représenter l'événement « Tlemcen Capitale de la Culture Islamique ». En effet, ce site qui bénéficie d'une dimension paysagère assez unique concentre aussi des ressources patrimoniales multiples. Par ailleurs, de par sa situation et superficie, le parc s'étend sur différentes communes, ce qui pourrait constituer un atout important pour un développement territorial.

\section{A. Le Parc National de Tlemcen, une plus-value pour le développement de projets de territoires durables}

Le Parc national de Tlemcen s'étend sur le territoire de 7 communes avec une superficie d'environ 8225 hectares -Tlemcen (731 ha), Mansourah (546 ha), Sabra (1682 ha), Ternie (3200 ha), Aïn Fezza (1535 ha), Beni-Mester (189 ha) et Aïn Gheraba (342 ha)
(Figure 2). Son altitude va de $670 \mathrm{~m}$ au niveau de l'Ourit à $1418 \mathrm{~m}$ à Djebel Koudia (Parc national de Tlemcen, 2009).

Il a été créé le 12 mai 1993 par le décret $n^{\circ} 93 / 117$ pour la conservation des faunes et des flores évoluant dans un microclimat humide (Décret $n^{\circ}$ $83-458$ du 23.07.1983). Sa richesse en matière de diversité biologique lui a valu en 2016 le classement de réserve internationale de biosphère par l'UNESCO (UNESCO, 2016).

En tant qu'établissement public à caractère administratif doté de la personnalité morale et de l'autonomie financière, le parc est géré par une direction nommée directement par le ministre de l'agriculture. Le décret $n^{\circ} 83-458$ du 23.07.1983 fixe le statut type des parcs nationaux et l'arrêté ministériel $n^{\circ} 358 / \mathrm{SPM} / \mathrm{DPPF} / 88$ précise les conditions d'intervention dans le parc. Un budget de fonctionnement est alloué annuellement par le ministère de tutelle. Un deuxième budget d'équipement peut être demandé en fonction des projets proposés par la direction du parc et approuvés par la tutelle.

Sur le plan paysager, le parc national de Tlemcen constitue une entité paysagère typique à l'échelle de la wilaya de Tlemcen. En plus de son intérêt écologique évident qui lui a permis d'être classé réserve de biosphère en 2016, il concentre aussi $70 \%$ des sites archéologiques arabo-musulmans (Sari, 2006) ainsi que des lieux culturels comme les mausolées qui continuent à attirer un nombre important de visiteurs (Sekkoum et Maachou, 2018).

\section{B. Diagnostic des ressources naturelles et cultu- relles du parc}

Comme déjà argumenté dans la justification du choix du site, le parc de Tlemcen regroupe de multiples ressources patrimoniales aussi bien naturelles que culturelles. La Figure 3 localise ces différentes ressources sur site.

\section{Les ressources naturelles du parc}

Le patrimoine naturel s'articule autour de quatre sites principaux (Tableau 3 ). Il s'agit des cascades d'El Ourit situées dans « le massif accidenté de Djebel Hanif », avec une superficie de 300 ha; des grottes féeriques de Beni-Add situées à une altitude 


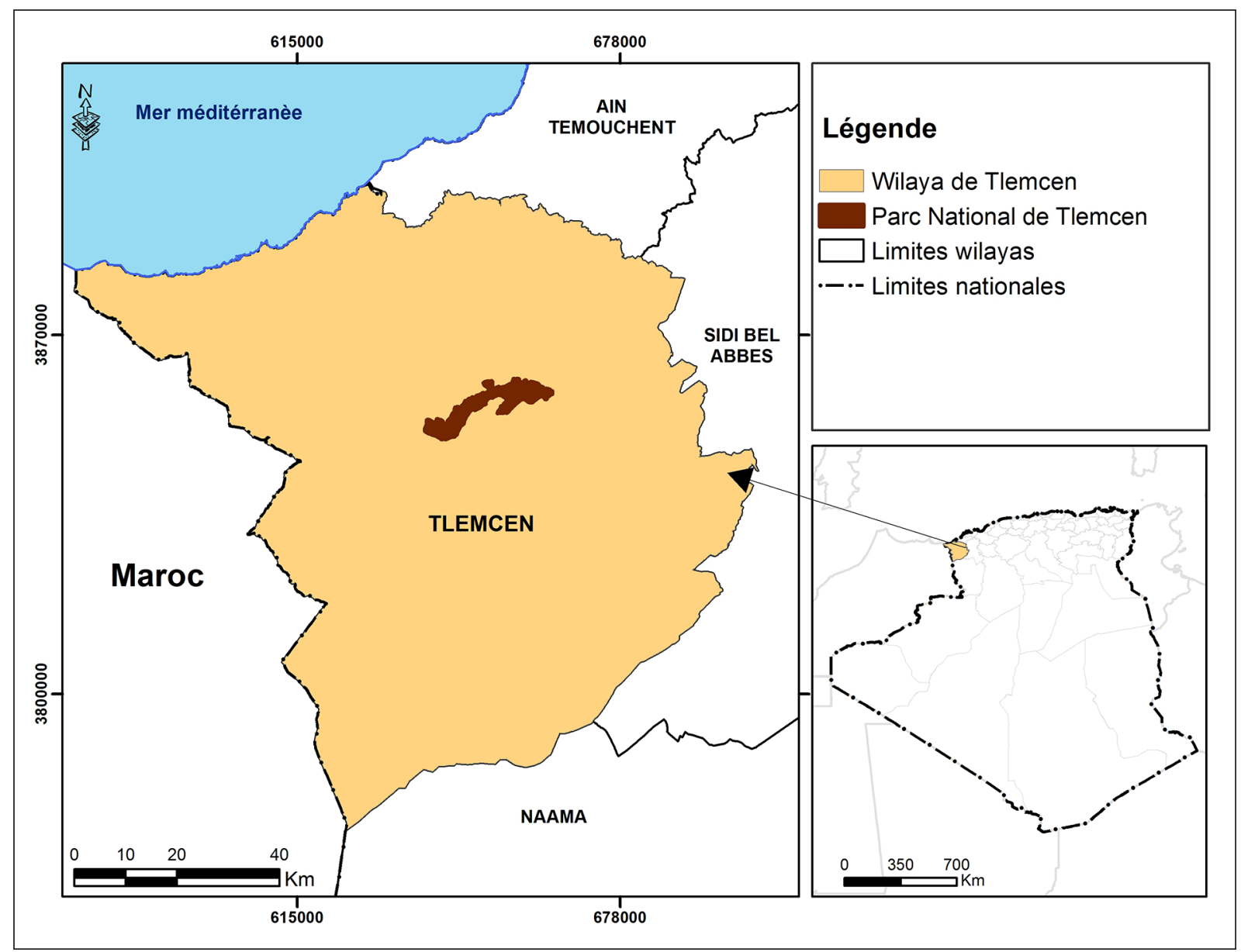

Figure 2. Situation du parc national de Tlemcen. Source : direction du parc de Tlemcen, 2017

de 1122 mètres. Ces grottes datent de plus de 65000 ans et constituent un musée naturel où stalagmites et stalactiques s'entrelacent en enfilades sur plus de $700 \mathrm{~m}$ de profondeur (Necissa, 2017). Des visites guidées y sont organisées de manière hebdomadaire. Le plateau de Lalla Setti qui s'étend sur 80 ha offre des vues panoramiques diverses. Le cimetière attenant au mausolée de Lalla constitue l'élément phare de ce plateau dans lequel plusieurs aménagements ont été introduits pour en faire un espace de détente et de récréation. La forêt de Tlemcen est, elle aussi, une forêt récréative plantée en 1890 sur environ 286 ha. Située à une altitude moyenne de 1096 mètres, elle est constituée principalement de pins d'Alep. À noter qu'en matière de flore, 953 espèces ont été recencées dont 39 protégées. La faune regroupe 305 espèces dont 61 protégées (Sekkoum et Maachou, 2018).

\section{Les ressources culturelles du parc}

Le patrimoine historique est composé de plusieurs sites historiques et archéologiques classés patrimoine national (Tableau 4). La mosquée de Sidi
Boumédiène, construite en 1328 avec son porche d'entrée monumental constitue le monument historique le plus important du parc (Khélifa, 2011). Le tombeau d'Abou Ishac et le mausolée de Sidi Abdallah attirent eux aussi les adeptes des zawiaa (équivalent de confrérie). Les ruines de Mansourah s'étalent sur environ 100 ha. Les vestiges des remparts, et en particulier le minaret de la mosquée qui atteint les 40 mètres de hauteur, comptent parmi les rares sites mérinides du Maghreb central classés en 1968 au patrimoine historique. La grande mosquée d'Agadir, fondée en 790, serait la première mosquée construite en Algérie (Khélifa, 2011). Seul le minaret qui émerge, avec ses 26 mètres de hauteur et quelques vestiges de ce qui constituait des murs, rappellent le rôle très important de Tlemcen dans le Maghreb central.

\section{Sur la mobilisation des acteurs concernés}

Pour valider ce travail d'investigation, nous avons procédé à des entretiens auprès de différents acteurs (institutions, communes, entreprises, mouvements associatifs) afin de comprendre la place qu'ils 
Le patrimoine comme levier de développement territorial.

Le cas des ressources patrimoniales du parc national de Tlemcen (Algérie)

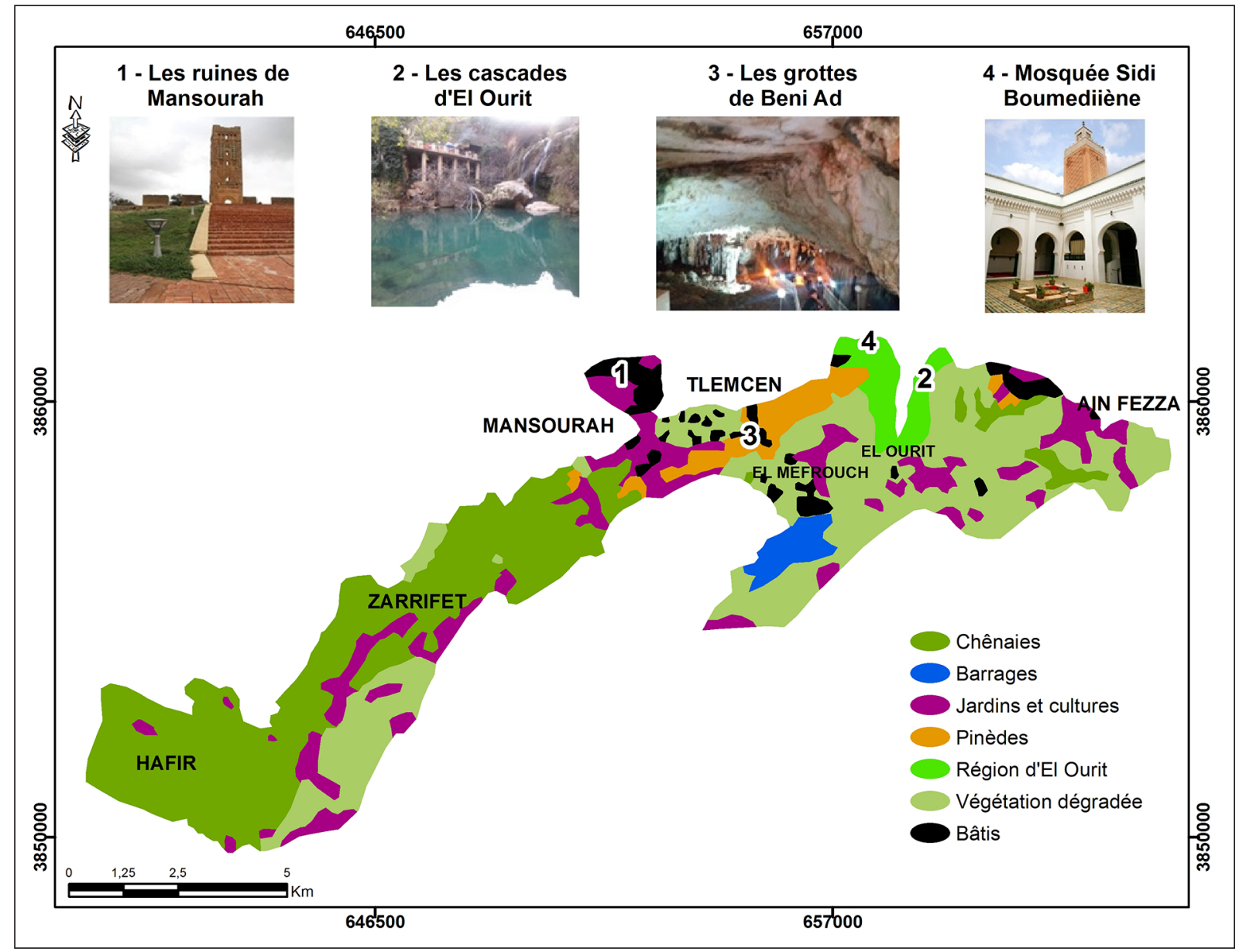

Figure 3. Potentialités patrimoniales du Parc National de Tlemcen. Source : Necissa, 2020

\begin{tabular}{|l|l|l|}
\hline Patrimoine naturel & Statut & Situation \\
\hline Cascades d'El Ourit & Paysage naturel & Commune de Tlemcen \\
\hline Plateau de Lalla Setti & Paysage naturel, parc d'attractions & Sud de la commune de Tlemcen \\
\hline Grottes de Beni Add & Paysage naturel & Commune de Ain Fezza \\
\hline Forêts de Zafir & Site naturel & Communes Ain Ghraba, Terry, Sabra \\
\hline
\end{tabular}

Tableau 3. Patrimoine naturel. Source : Necissa, 2020

\begin{tabular}{|l|l|l|}
\hline Patrimoine naturel & Statut & Situation \\
\hline $\begin{array}{l}\text { Sidi Boumedienne, Medersa, } \\
\text { Kobba et Bain }\end{array}$ & $\begin{array}{l}\text { Édifice historique J.O } \mathrm{n}^{\circ} 7 \mathrm{du} \\
\text { 23/01/1968 - Liste } 1900\end{array}$ & El Eubed, commune de Tlemcen \\
\hline Sidi Abdellah & Mausolée - Site classé 28/04/2013 & Commune de Tlemcen \\
\hline Sidi Abou Ishak & Mausolée - Site classé 20/12/1967 & Commune de Tlemcen \\
\hline $\begin{array}{l}\text { Mansourah, murailles et } \\
\text { minarets }\end{array}$ & $\begin{array}{l}\text { Édifice historique J.O }{ }^{\circ} 7 \mathrm{du} \\
\text { 23/01/1968 - Liste } 1900\end{array}$ & Commune de Mansourah \\
\hline Minaret d'Agadir & $\begin{array}{l}\text { Édifice historique classé } \\
01 / 02 / 1982\end{array}$ & Commune d'Agadir \\
\hline
\end{tabular}

Tableau 4. Patrimoine culturel. Source : Necissa, 2020 
accordent au patrimoine dans les perspectives de développement de leurs territoires. « Le diagnostic de territoire doit permettre la formulation d'un jugement sur la cohérence du territoire, mais aussi la mobilisation des acteurs. Il accompagne un changement dans le comportement des acteurs et dans les transformations de l'espace, dans une perspective de développement territorial » (Piveteau et Lardon, 2002).

Les différents acteurs concernés par la gestion du parc sont: (i) l'État représenté par les différentes directions (direction des forêts du parc, direction de la culture, et autres directions de wilaya). L'État intervient dans le cadre des programmes de restauration, étude d'inventaire, et de protection et mise en valeur des différents sites du parc. Son rôle consiste aussi à financer certaines études, (ii) les collectivités locales qui ont un rôle dans la valorisation touristique par la création d'événements et animations, (iii) les offices du tourisme qui sont les maîtres d'œuvre en matière de développement touristique. Ils coordonnent l'animation des loisirs, de la promotion, de la gestion ou de l'exploitation des équipements touristiques et enfin les associations citoyennes dont le rôle est le conseil et la sensibilisation de la société en matière de protection du patrimoine. Le Tableau 5 montre comment les différents acteurs impliqués dans ce projet de valorisation des ressources patrimoniales ont été interrogés.

\section{B. Les potentialités et contraintes des différents sites}

L'exploitation des données statistiques, les documents administratifs et techniques, les sorties sur sites nous ont permis de visualiser les potentialités et contraintes des différents sites comme suit:

\section{Les potentialités}

La lecture du territoire du parc a permis de mettre en évidence un potentiel patrimonial riche et varié dont certains monuments sont déjà classés. Ce patrimoine recouvre diverses valeurs, d'ordre culturel, spirituel, politique ou économique et plusieurs raisons justifient d'investir dans ce secteur. D'une manière générale, l'état de conservation est bon vu que ces sites ont fait l'objet de protection et mise en valeur à l'occasion de l'événement « Tlemcen Capitale de la Culture islamique en $2011 »$. Tous ces sites sont ouverts au public sauf le site d'Agadir.
Ce patrimoine peut donc être directement mobilisé dans la construction territoriale.

\section{Les contraintes}

La gestion très centralisée (up/down) semble être le plus grand handicap. À noter aussi le manque de coordination et de coopération entre les acteurs locaux eux-mêmes. La gestion budgétaire est elle aussi centralisée, ce qui retarde la programmation des projets de développement. Le budget de fonctionnement attribué annuellement par le gouvernement central ne suffit même pas à l'entretien quotidien du parc. Les acteurs impliqués dans la valorisation du parc n'ont pas une vision globale de développement de leur territoire et n'ont pas de compétences dans le marketing. Ainsi les circuits touristiques qui intègrent les différents sites historiques, culturels et naturels sont très peu nombreux et le manque de moyens de transport en commun rend l'accessibilité même à ces sites assez compliqués.

Ainsi, de manière générale, les principales actions et stratégies de développement adoptées sont ponctuelles et restreintes dans le temps, ce qui ne favorise pas une dynamique territoriale inclusive et pérenne.

\section{CAPITALISATION DES RÉSULTATS DE L'ÉVALUATION DES RESSOURCES PATRI- MONIALES DU PARC DE TLEMCEN}

Les résultats des analyses présentées précédemment sont reportés sur une grille spécifique d'évaluation. Cette analyse permet aussi de dégager des pistes de réflexion au sujet de nouvelles stratégies de valorisation économique du patrimoine.

\section{A. Indicateurs économiques}

Les indicateurs économiques paraissent plus favorables (Figure 4); sur onze indicateurs cités, deux indicateurs seulement ne sont pas pris en compte. Le premier est relatif aux retombées économiques sur le territoire et le second relatif à l'équilibre financier de la mise en valeur du patrimoine.

\section{B. Indicateurs sociaux}

Sur les onze indicateurs étudiés, un seul indicateur est bien pris en compte (Figure 5). Il s'agit de celui relatif aux organisations des manifestations culturelles liées au patrimoine tels les festivals 
Le patrimoine comme levier de développement territorial.

Le cas des ressources patrimoniales du parc national de Tlemcen (Algérie)

\begin{tabular}{|c|c|c|}
\hline \multirow{13}{*}{ État } & Catégories d'acteurs & Informations demandées \\
\hline & Ministère de l'Agriculture & \multirow{2}{*}{$\begin{array}{l}\text { Enjeux stratégiques de valorisation du } \\
\text { patrimoine engagés ou envisagés }\end{array}$} \\
\hline & Ministère de la Culture & \\
\hline & $\begin{array}{l}\text { Propriétaires fonciers, promo- } \\
\text { teurs et offices du tourisme }\end{array}$ & Investissements pour les touristes \\
\hline & Direction des forêts & \multirow{9}{*}{$\begin{array}{l}\text { Programme de restauration, étude d'inventaire } \\
\text { et de protection et mise en valeur des différents } \\
\text { sites du parc. } \\
\text { Projets de développement du parc. }\end{array}$} \\
\hline & Direction de l'Agriculture & \\
\hline & Direction de l'Urbanisme & \\
\hline & Direction de la Construction & \\
\hline & Direction du Tourisme & \\
\hline & Direction de l'Hydraulique & \\
\hline & Direction de l'Environnement & \\
\hline & Direction de la Culture & \\
\hline & $\begin{array}{l}\text { Direction de la Planification et } \\
\text { de l'Aménagement du territoire }\end{array}$ & \\
\hline Acteurs locaux & $\begin{array}{l}\text { Autorités publiques : les sept } \\
\text { communes de Tlemcen, Man- } \\
\text { sourah, Terne, Ain Fezzan, } \\
\text { Sabra, Ain Chorba, Beni Mestre }\end{array}$ & $\begin{array}{l}\text { Recettes générées par le site (billeterie, bou- } \\
\text { tique-librairie, visites guidées, événements ...), } \\
\text { emplois (salariées, bénévoles, saisonniers), } \\
\text { financements (public, privé...) }\end{array}$ \\
\hline \multicolumn{2}{|c|}{ habitants et associations civiles } & $\begin{array}{l}\text { Sensibilisation de la société en matière de pro- } \\
\text { tection du patrimoine }\end{array}$ \\
\hline
\end{tabular}

Tableau 5. Acteurs du parc national de Tlemcen. Source : Necissa, 2020

folkloriques et autres. Il s'agit donc d'impliquer plus largement les populations concernées à travers des ateliers de sensibilisation à la valorisation des ressources de leur territoire. Les populations locales doivent devenir des acteurs à part entière dans le développement de leur territoire.

\section{Gouvernance et gestion}

Sur les onze indicateurs étudiés, sept ne sont pas pris en compte, quatre sont moyennement pris en compte (Figure 6). Ces résultats très critiques mettent en évidence l'importance de l'existence de structures locales et nationales, réellement consacrées à la valorisation des ressources patrimoniales. La capacité à mobiliser des aides techniques et financières internationales est elle aussi primordiale. Il s'agit aussi de promouvoir une certaine autonomie des collectivités locales et d'encourager une gestion plus « bottom/up » et inclusive qui favorise la participation de tous les acteurs concernés tels les mouvements associatifs.

Les résultats du profil en radar général montrent que l'indicateur le plus élevé est celui de la dimension économique (Figure 7), alors que l'indicateur de gestion et gouvernance reste très faible. L'AMC a donc été très fructueuse et donné un ensemble d'éléments et d'indicateurs intéressants qui permettent de discuter les mesures envisagées et de définir les choix stratégiques à adopter en matière de développement territorial.

Ces mesures concerneraient en priorité le volet de participation effective des populations locales, en tant qu'acteurs actifs dans le processus de prise de décision. Il s'agit aussi d'attirer et d'encourager les investissements dans le domaine du tourisme responsable.

Alléger la fiscalité pour les métiers du terroir et autres artisans pourrait contribuer à favoriser la construction d'une image plus attractive et compétitive à travers la multiplication des opérations de labellisation. Plusieurs écrits ont montré que la mise en place de projets autour du patrimoine favorise la promotion et la valorisation de l'identité du territoire (Virassamy, 2002 ; Bonerandi, 2005 ; Landel et Senil, 2009; Pin et al., 2013 ; Vernières, 2015). Cette première étape dans l'expression du 


\section{Dimension économique}

1. Attractivités du territoire pour ses activités dans le domaine du patrimoine.

10. Retombées économiques surle territoire.

\section{Coût de la gestion} touristique pour la collectivité accueil, sécurité, informatio...

\section{Activité des sites culturels : droits}

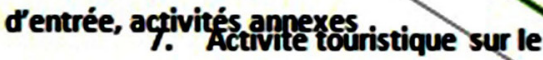
territoire : entrées, nuitées, dépenses moyennes, durée de séjour
2. Activités économiques en place liées au patrimoine : droits d'entrée, transport touristique.

\section{Dépenses publiques} associées au patrimoine

4. Dépenses d'entrée des résidents dans le patrimoine

\section{Dépenses de} maintenance et d'entretien pour l'accueil des visiteurs

\section{Investissements dans}

Paccueil des visiteurs

Figure 4. Radars de la dimension économique. Source : Necissa, 2020

\section{Dimension sociale}

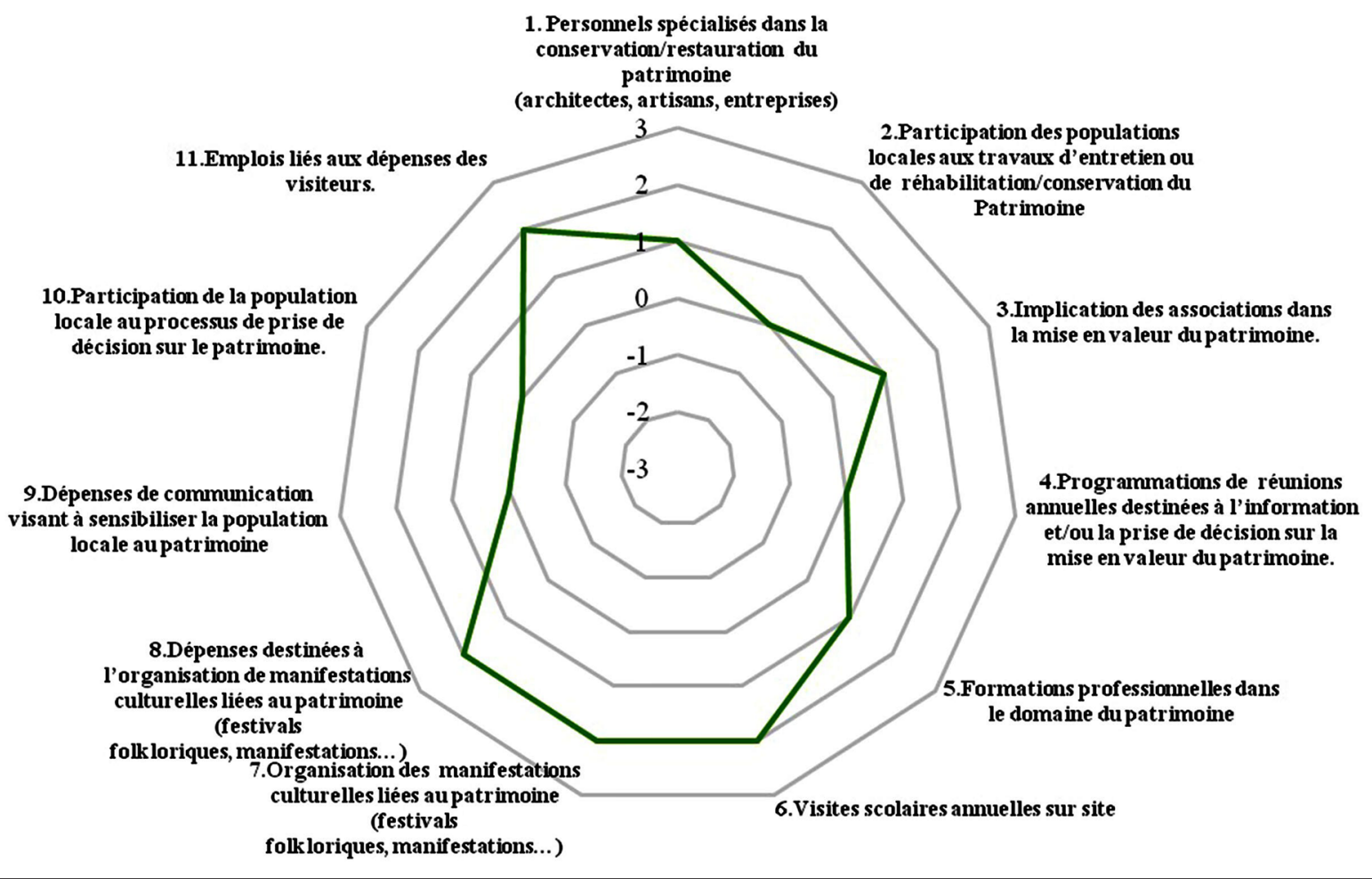

Figure 5. Radar de la dimension sociale. Source : Necissa, 2020 
Le cas des ressources patrimoniales du parc national de Tlemcen (Algérie)

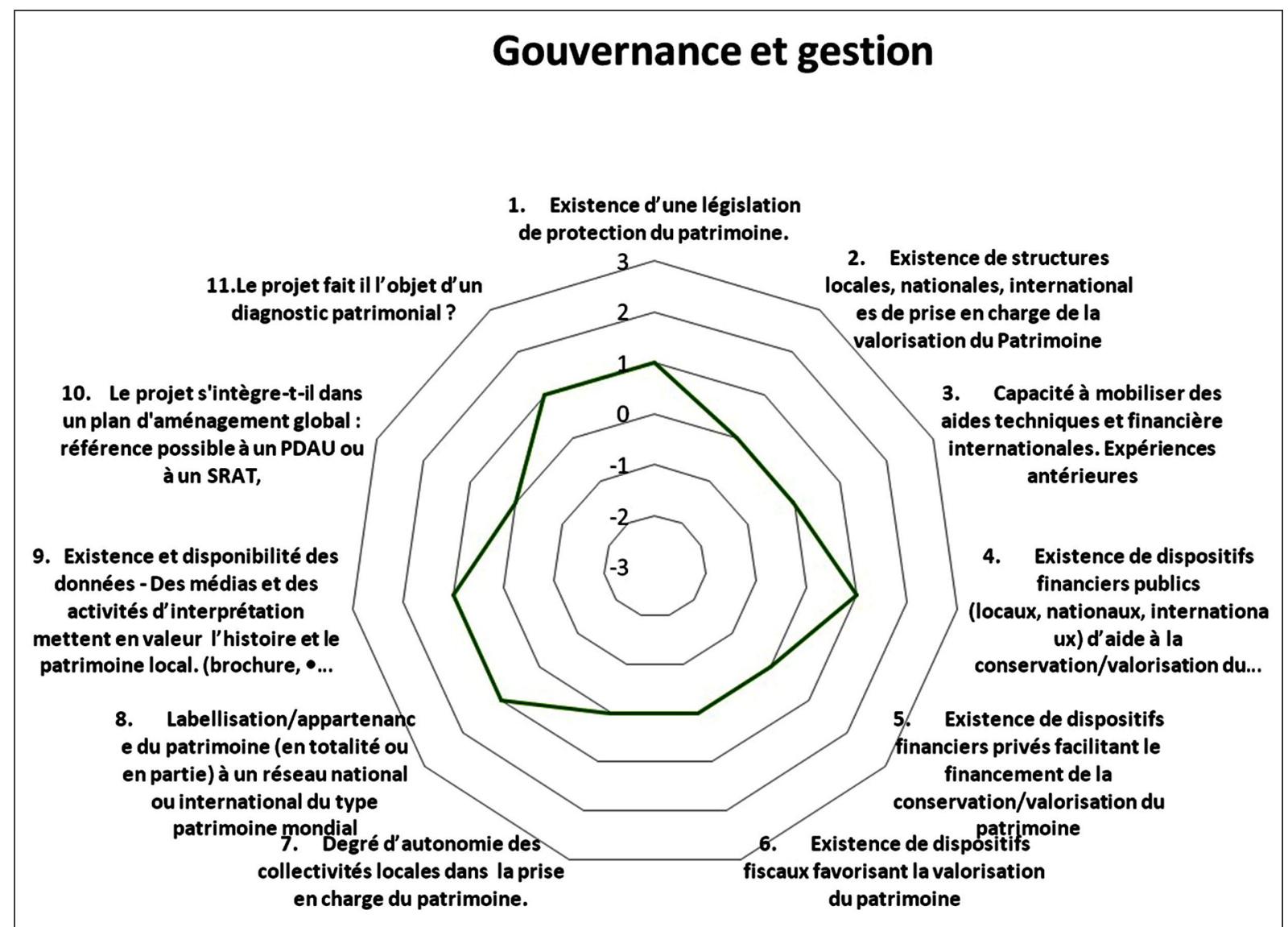

Figure 6. Radar de la Gouvernance. Source : Necissa, 2020

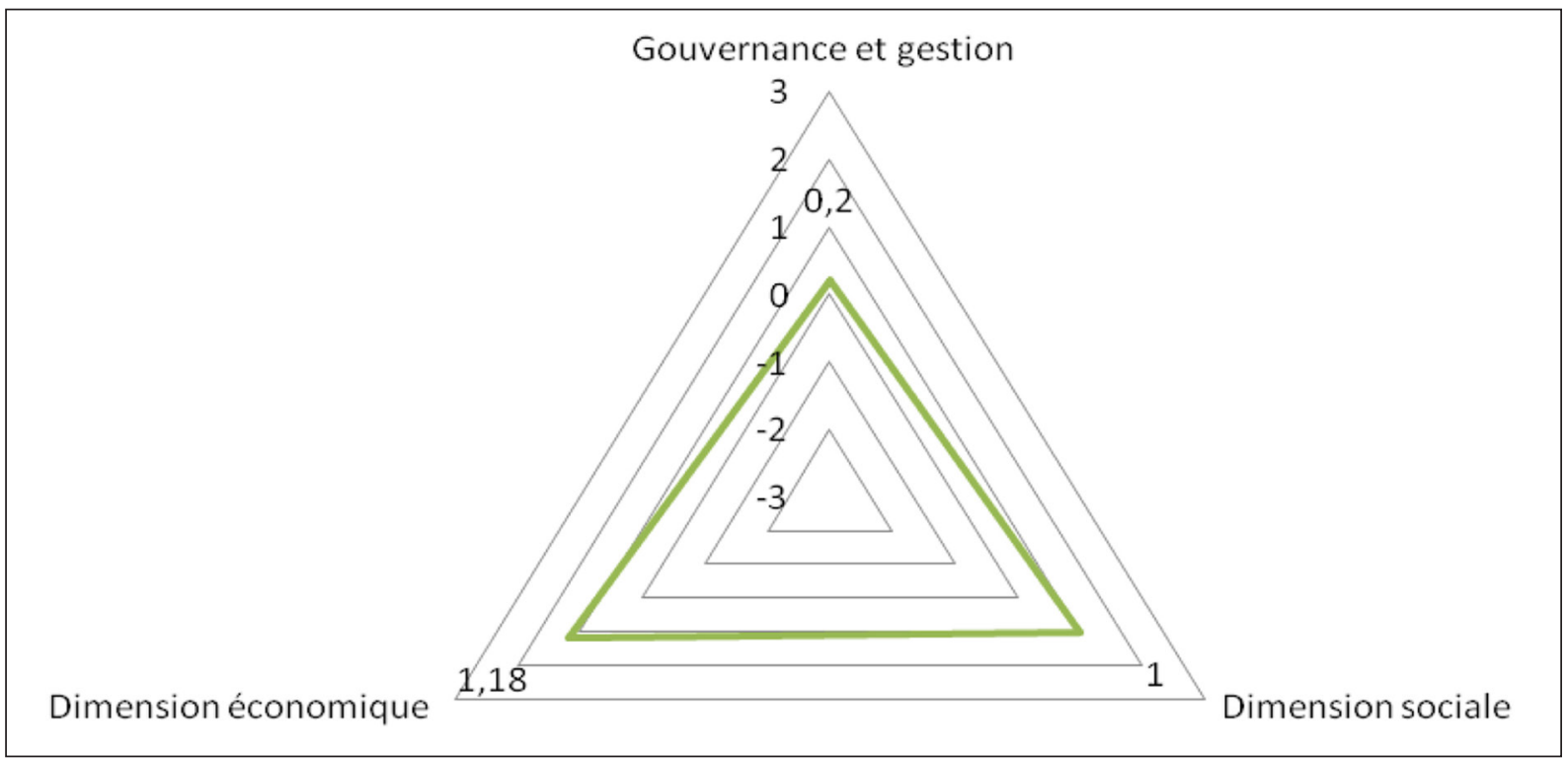

Figure 7. Profil général. Source : Necissa, 2020

changement souhaité, pourrait donner un nouvel élan de développement, plus en adéquation avec les ressources identifiées.

Par ailleurs, une bonne stratégie de marketing territorial permettrait de mobiliser toutes les ressources patrimoniales du parc pouvant jouer un rôle dans la valorisation du territoire. En effet, différents au- teurs ont montré comment le marketing territorial pouvait contribuer à une transformation accélérée du territoire et à accroître l'attractivité de ce dernier en vue d'un développement territorial harmonieux aux yeux de l'ensemble des parties prenantes (Chamard et Schlenker, 2017). En effet, au-delà de la communication, le marketing territorial apporte un ensemble de repères (méthodes, techniques, outils, 
etc.) qui, mis ensemble, permettent de faciliter l'élaboration d'une stratégie collective en faveur de l'attractivité territoriale. L'intérêt de cette démarche réside également dans sa capacité à assembler des acteurs différents et aussi des élus, des décideurs et des techniciens (Figure 8).

Le diagnostic a aussi permis de montrer que les ressources patrimoniales du parc de Tlemcen sont porteuses de nombreuses valeurs. Ces ressources patrimoniales sont par nature plus fragiles que les autres types de ressources. Il est nécessaire d'envisager une gestion appropriée de leur valorisation. On peut postuler qu'un nouveau type de gouvernance sera nécessaire dans ce type de gestion, qui devra adapter ses réponses, pour pérenniser la ressource et pérenniser le développement lié à cette ressource (Telaidjia, 2016). Le patrimoine doit être géré d'une manière sensible, intelligente et dynamique (Gravari-Barbas, 2001), il s'agit d'une gouvernance patrimoniale qui considère le patrimoine comme l'image du territoire qui participe à sa construction et son développement.

\section{CONCLUSION}

Le présent article avait pour objectif d'élaborer et tester un outil d'évaluation de la capacité de contribution des ressources patrimoniales au développement territorial. Les résultats montrent que le patrimoine du parc national de Tlemcen représente un potentiel de création de richesse et qu'il peut être mis en valeur.

Les résultats ont aussi permis d'identifier certaines lacunes et faiblesses liées à la gestion et l'aménagement des sites, au financement des opérations d'entretien et de mise en valeur du patrimoine ainsi que l'absence de coordination entre les différents acteurs. Le système de prise de décision est très centralisé et les acteurs locaux sont réduits au rôle d'exécutants des directives reçues de l'administration centrale. Leur vulnérabilité est d'autant plus exacerbée par le fait qu'ils dépendent financièrement des subsides de l'État. Le mouvement associatif est lui-même émergent et ses actions sont très ponctuelles et restreintes.

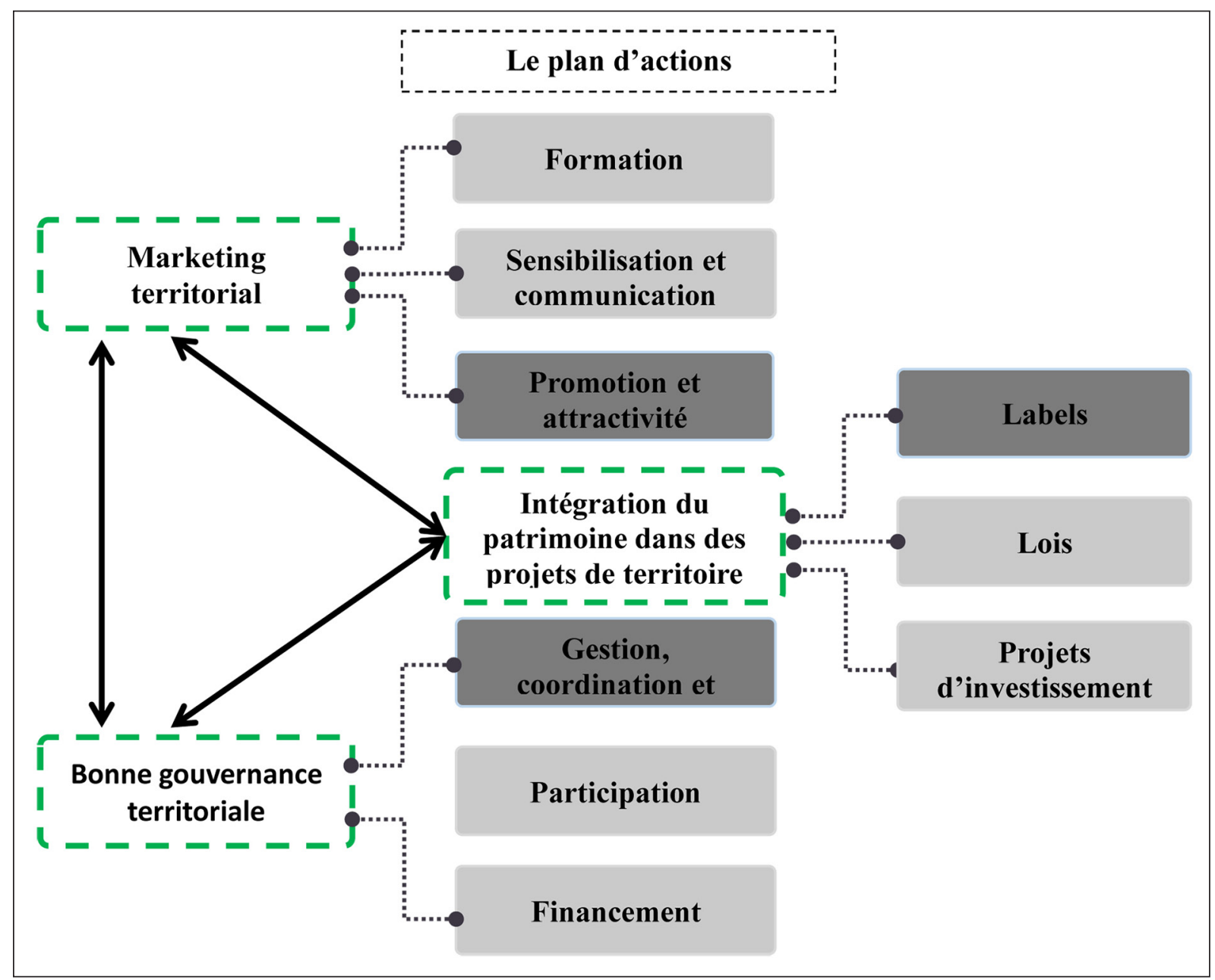

Figure 8. Plan d'actions pour la mise en valeur du patrimoine du parc. Source : Auteurs, 2020 
Le cas des ressources patrimoniales du parc national de Tlemcen (Algérie)

Pour répondre à notre objectif, la démarche proposée doit prendre en charge l'amélioration de la gestion et la gouvernance à travers le renforcement du partenariat public-privé, le développement de la coopération intercommunale et la coordination entre les différents secteurs et acteurs. La participation de la société civile et la mise en place d'outils contextualisés et inclusifs d'aide à la décision contribueraient à renforcer l'adhésion des habitants aux projets de développement de leurs territoires et en assurer la pérennité.

\section{NOTES}

${ }^{1}$ Sous-titre emprunté à Dominique Mataillet, «Tlemcen, la Grenade africaine », Jeune Afrique, 28 avril 2010.

\section{LISTE DES ACRONYMES}

AMC : Analyse multicritère

PAW : Plan d'aménagement de wilaya

PAT : Programme d'action territorial

PEP : Pôle d'Economie du Patrimoine

SDAL : Schéma directeur d'aménagement du littoral

SNAT : Schéma National d'Aménagement du Territoire

SRAT : Schéma Régional d'Aménagement du Territoire

\section{BIBLIOGRAPHIE}

Abichou, H. (2009). La valorisation du patrimoine vecteur de développement local : quelles retombées économiques et quel dispositif institutionnel en Tunisie? Cas du sud-est tunisien. Thèse de Doctorat, École doctorale économie de gestion Montpelier, 309p.

Amougou, E. (2004). La question patrimoniale : de la patrimonialisation à l'examen des solutions concrètes. Ed L'harmattan, $286 \mathrm{p}$.

Audrerie, D. (2003). Questions sur le patrimoine. Confluences édition, Collections stèle, $119 \mathrm{p}$.

Baudelle, G., Guy, C. \& Mérenne-Schoumaker, B. (2011). Le Développement Territorial En Europe Concepts, Enjeux Et Débats. 281 Pages.

Barrère, C., Bonnard, Q. \& Delaplace, M. (2014). Valorisation des patrimoines et dynamique régionale. Territoire en mouvement. Revue de géographie et aménagement [En ligne], consulté le 05 août 2020. URL : http://journals.openedition.org/tem/2278

Bonerandi, E. (2005). Le recours au patrimoine, modèle culturel pour le territoire? Géocarrefour, 91-100.

Berger, A, Chevalier, P., Cortes. G. \& Dedeire, M. (2010). Patrimoines, héritages et développement rural en Europe. L'Harmatan, Logiques sociales, Paris, 250p.

Chamard, C. et Schlenker, L. (2017). La place du marketing territorial dans le processus de transformation territoriale. Gestion et management public, 6/1(3), 41-57.

Di Méo, G. (1995). Les nouvelles contradictions du territoire / New territorial contradictions. Géocarrefour, 169-175.

Di Méo, G. (1995). Patrimoine et territoire, une parenté conceptuelle. Espaces et Sociétés, 78, 15-34.

Doré, D. (2005). L'opérationnalisation des principes du développement durable au sein des processus d'analyse de projets au Saguenay-Lac-Saint-Jean: obstacles et piste de solution. Thèse de Doctorat en développement régional, Université du Québec à Chicoutimi en vertu d'un protocole d'entente avec l'Université du Québec à Rimouski, Chicoutimi, Québec, 714 p.

Dumas, L. (2014). Outil d'évaluation de projets potentiels de développement des ressources naturelles et du territoire des régions ressources du Québec. Maîtrise en Environnement de l'Université de Sherbrooke, 111p.

El Ansari R. (2008). Le Développement régional face aux disparités socio-économiques au Maroc. Thèse de Doctorat en Urbanisme, Aménagement et Politiques urbaines, Institut d'Urbanisme de Paris, Université Paris XII -Val-de-Marne, 379 p.

Fagnoni, E. (2013). Patrimoine versus mondialisation? Revue Géographique de l'Est [En ligne], 53/34, http://journals.openedition.org/rge/5048

François, H., Hirczak, M. et Senil, N. (2006). Territoire et patrimoine : la co-construction d'une dynamique et de ses ressources. Revue d'Économie Régionale \& Urbaine, décembre(5), 683-700. doi:10.3917/ reru. 065.0683

François, H., Hirczak, M. \& Senil, N. (2010). La dimension patrimoniale des ressources territoriales. Quel rôle dans les trajectoires de développement ? In « Patrimoines et héritages pour le développement rural en Europe », L'Harmattan, 21-44.

François, H., Hirczak, M. et Senil, N. (2013). De la ressource à la trajectoire : quelles stratégies de développement territorial ? Géographie, économie, société, 15(3), 267-284.

Gautier, J. (2005). Développement d'un outil d'aide à la décision environnementale durable pour une grande ville. Mémoire de maîtrise ès sciences appliquées (génie civil), École polytechnique de Montréal, Montréal, Québec, 107 p.

Ghomari, F. (2007). La médina de Tlemcen: l'héritage de l'histoire. Web journal on cultural patrimony, 2(1), 11-28.

Gravari-Barbas, M. (1996). Le « sang » et le « sol» : le patrimoine, facteur d'appartenance à un territoire urbain. Géographie et cultures, 20, 55-67.

Idir, M.S. (2013). Valorisation du patrimoine, tourisme et développement territorial en Algérie: cas des ré- 
gions de Béjaïa en Kabylie et Djanet dans le Tassili n'Ajjer. Thèse de Doctorat, Laboratoire PACTE dans l'École Doctorale Sciences Économiques, Université de Grenoble, 375p.

Khelifa, A. (2011). Tlemcen, capital du Maghreb central. Édition Colorset, Alger, 421p.

Lacour, C., Leloup, F. \& Moyart, L. (2014). Introduction. Culture, patrimoine, savoirs : facteurs dynamiques de développement. Revue d'Économie Régionale \& Urbaine, décembre(5), 785-799.

Landel, P. Mao, P. \& Vidal, L. (2007). Le diagnostic de territoire et la création d'activités touristiques à partir du patrimoine.in tourisme et patrimoine, un moment du monde. Presse de l'Université d'Angers, pp.175-186.

Le Galès, P. (2010). Gouvernance. In Laurie Boussaguet éd., Dictionnaire des politiques publiques: 3e édition actualisée et augmentée (pp. 299-308). Paris: Presses de Sciences Po.

Leloup, F., Moyart, L. \& Pecqueur, B. (2005). La gouvernance territoriale comme nouveau mode de coordination territoriale ? Géographie, économie, société, 7(4), 321-332.

MATE (2008). La mise en œuvre du Schéma National d'Aménagement du Territoire (SNAT) 2025, rapport préliminaire. Ministère de l'Aménagement du Territoire et de l'Environnement, Alger, 28p.

MATE (2010). Loi $\mathrm{n}^{\circ} 10-02$ du 29 juin 2010, portant approbation du Schéma National d'Aménagement du Territoire, Ministère de l'Aménagement du Territoire et de l'Environnement, Alger, 110 p. www.joradp.dz.

Marçais, G. (1950). Tlemcen. Éditions H. Laurens, collection les Villes d'Art Célèbres, 104p.

Necissa, Y. (2001). Le patrimoine comme outil de développement territorial cas de la wilaya de Médéa. Mémoire de magister, École polytechnique d'architecture et d'urbanisme, EPAU, Alger, 200p.

Necissa, Y. (2017). La valorisation économique du patrimoine. Thèse de Doctorat en Sciences, École polytechnique d'architecture et d'urbanisme, EPAU, Alger, 270p.

Pecqueur, B. (2006). Le tournant territorial de l'économie globale. Espaces et sociétés, 124-125(1), 17-32.

Pin. B., Rode, S. \& Servain, S. (2013). Processus de construction d'une ressource territoriale valorisant le patrimoine naturel et culturel en Loire tourangelle. VertigO - la revue électronique en sciences de l'environnement [En ligne], Hors-série 16, consulté le 05 août 2020. URL : http://journals.openedition. org/vertigo/13713

Pupin, V. (2008). Les approches patrimoniales au regard de la question de la prise en charge du monde. Thèse de Doctorat, Institut des Sciences et Industries du vivant et de l'environnement de Paris, 302p.

René, D., \& Marçais, G. (1952). Tlemcen (Les Villes d'art célèbres). In Syria, Tome 29 fascicules 3-4, 367p.

Sari, D. (2006). Tlemcen: la cité-patrimoine à sauvegarder : la Tachfinya à reconstruire impérativement. Éditions ANEP, Université du Michigan, 117p.

Senil, N. (2011). Une reconstruction de l'espace-temps : approche croisée des processus de patrimonialisation et de territorialisation dans les territoires ruraux en France et au Maroc. Thèse de Doctorat, Université Joseph Fourier, 562p.

Sekkoum, S., \& Maachou, H. (2018). Le parc national de Tlemcen (Algérie) : un potentiel touristique sous-exploité. Études caribéennes [En línea], 3940 |, Publicado el 15 julio 2018, consultado el 04 agosto 2020. DOI : https://doi.org/10.4000/etudescaribeennes. 12450

Vanderpooten, D. (2009). Introduction à 1'aide multicritère à la décision. In R. Faure, B. Lemaire, C. Picouleau (eds), Précis de recherche opérationnelle, 6ème édition Dunod, 419-441.

Vernières, M. (2015). Le patrimoine : une ressource pour le développement. Techniques Financières et Développement, 118(1), 7-20.

Virassamy, C. (2002). Les pôles d'économie du patrimoine. Documentation Française - Coll. Territoires en mouvement, $90 \mathrm{p}$.

Coordonnées des auteurs :

Yamina NECISSA

Institut d'architecture et d'urbanisme de Blida Algérie aminanecissa65@hotmail.com

\section{Naima CHABBI CHEMROUK} professeur EPAU Algérie

Laboratoire de Recherche architecture et environnement EPAU Ecole polytechnique d'architecture et d'urbanisme Alger n.chemrouk@epau-alger.edu.dz 